\title{
Why motor simulation cannot explain affordance perception
}

\begin{abstract}
According to several authors in psychology and neurosciences, our ability to perceive affordances is subtended by motor simulation mechanisms. Such mechanisms provide dynamic representations of feasible actions, thus enabling to scale the surrounding structures on the behavioural repertoire and capacities supported by our body. This attractive hypothesis has been taken up in robotics, to build intelligent systems able to determine in advance if a given action would be successful given the current state of the environment and their own skills.

Several arguments however suggest that the motor simulation framework is not sufficient to explain affordance perception: $(i)$ it rests on a misunderstanding of what affordances are: not actions that are currently feasible, but actions that are possible; (ii) it is computationally unrealistic: motor simulation is too costly in terms of computational resources to explain how one can access prospectively to actions that are potentiated by surrounding structures; (iii) it only covers the part of the perceptual field within the scope of our attention, but the affordances we perceive do not reduce to the object or state of affairs our attention is focused on at time $t$; (iv) it can only work if a first layer of affordances is available: motor simulation cannot explain affordance perception, because its very functioning presupposes such perception. Other mechanisms must consequently be hypothesized.
\end{abstract}

\section{Keywords}

Motor simulation, mental imagery, sensorimotor emulation, affordance, visual perception, robotics.

\section{Introduction}

Motor simulation is undoubtedly a successful concept. The range of cognitive skills it could be involved in has considerably expanded since its first use in the field of motor control theories, and most researchers nowadays assume that it plays a prominent role in processes as diverse as motor control and motor planning (Johnson, 2000; Jeannerod, 2001), mindreading (Gallese \& Goldman, 1998; Decety \& Stevens, 2009), language processing (Pulvermüller, 2005; Wheeler \& Bergen, 2010), imitation-based skill learning (Goldman, 2005) or tool-use recognition (Johnson-Frey, 2004; Witt el al., 2010).

The range of cognitive skills covered by motor simulation has still expanded in past years with the claim that motor simulation mechanisms can also explain how one can develop perceptual knowledge of affordances, i.e. behavioural opportunities potentiated by the objects and structures of the environment. Garbarini \& Adenzato (2004), for instance, state that the representation of objects 'in relation to the type of action that [they afford] an interacting subject' proceeds from 'a mechanism of as-if neural simulation: while observing an object, the neural system is activated as-if the observer were interacting with it'.

In the field of neuropsychology, this theoretical proposition - that we will refer hereinafter as the simulation theory of affordance perception (STAP) - was first formulated by Marc Jeannerod (Jeannerod 1994, 2001, 2003; Jeannerod et al, 1995). 
According to Jeannerod, the function of motor simulation is "not only to shape the motor system in anticipation to execution, but also to provide the self with information on the feasibility and the meaning of potential actions' (Jeannerod, 2001). Following Ungerleider \& Mishkin (1982) and Goodale \& Milner (1992), Jeannerod distinguishes two visual information processing pathways: (i) a semantic pathway, processing object attributes for action-independent tasks, as properties description or naming; and (ii) a pragmatic pathway, building action-related representations, i.e. 'representing objects as goals for action' (Jeannerod, 2003). The pragmatic pathway enables the brain to represent the action possibilities available at a given moment. By simulating actions directed to objects, e.g. reach or grasp actions, it is able to determine in advance if such actions can be done.

The core principle of such an idea is not new. Kenneth Craik (1943), known to have laid the conceptual foundations of the computo-symbolic approach to cognition and mental model framework, already considered simulation a basic means for cognitive systems to model processes taking place in their environment, predict future events and organize their behaviour in a proactive way. In Craik's opinion, the better way to predict the outcomes of a process is to "play" it in advance. Yet our brain does not have the resources or time required for a full-scale achievement, as a result only a simplified model of the real process is played and it is played virtually, through pure symbolic means. 'If the organism carries a "small-scale model" of external reality and of its own possible actions within its head, it is able to try out various alternatives, conclude which is the best of them, react to future situations before they arise, utilise knowledge of past events in dealing with the present and future, and in every way to react in a much fuller, safer, and more competent manner to the emergencies which face it.' (Craik, 1943: 61)

Since Jeannerod's early description of pragmatic representations, STAP hypothesis has been refined and defended in more or less strong forms by several researchers in psychology and neurosciences (de'Sperati \& Stucchi, 1997, 2000; Tucker \& Ellis, 1998; Gallese, 2000; Ellis \& Tucker, 2000; Tucker \& Ellis, 2001, 2004; Hesslow, 2002; Grush, 2003, 2004, 2007; Garbarini \& Adenzato, 2004; Tipper et al., 2006; Cisek, 2007; Coello \& Delevoye-Turrell, 2007; Witt \& Proffitt, 2008; Delevoye-Turrell et al., 2010; Caligiore et al., 2010; Sinigaglia \& Rizzolatti, 2011; Ellis et al., 2013) and in the field of robotics (Möller, 1999; Ziemke el al., 2005; Hesslow \& Jirenhed, 2007; Hoffman, 2007; Erdemir et al., 2008; Schenck, 2009; Schenck et al., 2012). STAP has especially been used to explain perceptual access to affordances related to manual prehension process: how reachability and graspability of objects located in the peripersonal space can be perceived. In addition, it was radicalized by authors as Garbarini \& Adenzato (2004), with the claim that motor simulation is the only way to develop knowledge of action possibilities made available by objects.

Needless to say, STAP hypothesis addresses important theoretical issues. The ability to perceive what actions are achievable in the environment and to categorize objects accordingly is undoubtedly an essential cognitive skill in ecological context: to react correctly to situations, we must be able to anticipate what we can and cannot do given the available surrounding structures. We must consequently possess a precise knowledge of our behavioural capacities and attunements of those capacities to situations, objects and states of affairs.

The problem is however to determine if motor simulation is requested to develop such knowledge. When considering the core function of the simulation mechanism, STAP's claim appears justified: a virtual achievement of actions (Decety, 1996a; Jeannerod, 2001) seems to be a straightforward means to evaluate their feasibility and anticipate 
their consequences in a given context, i.e. to represent and evaluate counterfactuals, and categorize objects accordingly.

Yet this claim is less obvious than it seems when taking carefully into account the empirical data available and the nature of what we perceive when we perceive affordances. STAP probably looks convincing at first sight because one tends to think that affordances being merely possible actions (we are not currently performing those actions, we could perform them), they necessarily need to be simulated to be conceived or perceived, i.e., to have a cognitive reality. But think about it: do we really have to simulate the actions we could perform with this or that structure to perceive such structure as enabling those actions? When we perceive a chair - an object affording sitting behaviour - is our brain playing covertly the action of sitting on this chair? In the following, I will present several arguments that clearly challenge such claims and weaken STAP's explanatory significance: $(i)$ it rests on a misunderstanding of what affordances are: not actions that are currently feasible, but actions that are possible; (ii) it is computationally unrealistic: motor simulation is too costly in terms of computational resources to explain how one can gain knowledge of actions that are potentiated by surrounding structures; (iii) it only covers the part of the perceptual field within the scope of our attention, but the affordances we perceive do not reduce to the object or state of affairs our attention is focused on at time $t$; $(i v)$ it can only work if a first layer of affordances is available: motor simulation cannot explain affordance perception, because its very functioning presupposes such perception ${ }^{1}$.

These arguments do not demonstrate that STAP is false in itself; they only show that its scope must be reconsidered. Motor simulation is probably centrally involved in situations where an explicit evaluation of action feasibility is engaged (that is, situations where what can or will be done is for the main part already decided), but it cannot account for possibilities for which such assessment is lacking, i.e. the greater part of the affordances we are aware of. Other mechanisms must consequently be hypothesized.

It should be noted that it is not the aim of this paper to propose any model of such mechanisms. Our objective is to demonstrate why STAP is not enough to explain affordance perception, it is not to explain how affordances are perceived. In that respect, our contribution is threefold: (a) we propose a more limited scope for STAP, which in our view makes its claim more consistent; (b) we propose to distinguish between (here and now) feasible actions and possible actions, which can help to clarify the current debate on motor simulation and affordance; (c) we propose a new perspective on the function of motor simulation in the cognitive mechanisms underlying the awareness of what can be done in the environment.

\section{Affordances cannot be reduced to currently available action possibilities}

A first argument that can be made against STAP relates to the meaning of the concept of affordance itself, and posits that affordance, as defined by Gibson and his followers,

\footnotetext{
1 The hypothesis that motor simulation is necessary for affordance perception is also challenged by neuropsychological data. For instance, Negri et al. (2007) have described post-stroke apraxic patients who are severely impaired in using or mimic the use of common objects - what suggests that brain mechanisms responsible for motor control are impaired - but can still recognize them and identify their functional properties, and other patients showing symmetric patterns of performances. As the authors explain, those data suggest 'that the processes subserving object use are not necessary in order to recognize and understand objects, and vice versa'. Although important, our claim in this paper will however not lean on such data, preferring instead more fundamental issues.
} 
is simply not compatible with the use made by STAP. This claim can be supported by the two following statements: (1) affordances are not representations; (2) affordances refer to actions that are possible in principle for a referent system, not to actions that are currently feasible (can be performed here and now) for this system.

A constitutive aspect of STAP is the challenging of Gibson's conception of affordance perception as direct, i.e. not mediated by representational content or inference mechanism. According to STAP, the perceptual access to actions afforded by surrounding structures is mediated by dynamic motor representations relying on the same neural processes as effective motor execution. As explained by Möller, 'the "utility" of objects is not directly "offered" by the external world, but determined by the generation of sensorimotor hypotheses based on the sensory input' (Möller, 1999). STAP thus 'replaces the direct perception of affordances by a mental simulation process based on internal models' (Schenck, 2009), and adds a third term to the relation between action and perception (Jeannerod \& Gallagher, 2002; Garbarini \& Adenzato, 2004).

However, the question arises as to whether STAP supporters and Gibson are really talking about the same thing. Can we still speak of affordances once admitted that they are (internally) represented?

A point frequently made by Gibson and his followers is that affordances are true physical (although relational and dispositional) properties of the physical reality (Gibson, 1986: 140-141; Turvey, 1974, 1992). Their existence does not depend on the individual's current needs, or attention and perception processes (Gibson, 1986: 138139), and they cannot be equated to a kind of mental representation, e.g., instrumental or functional representation (Chemero \& Turvey, 2007). The use STAP supporters, typically authors like Ellis \& Tucker (2000), make of the notion of affordances is consequently not gibsonian: undoubtedly, the facilitation effects they observe suggest that in a way or another 'seen objects potentiate a range of actions associated with them', but calling affordances the representation of the motor patterns that are automatically activated during the visual processing of e.g. reachable or graspable objects is a misunderstanding (see Michaels, 2003: 136).

However, this is not the point I would like to insist on here. In my view, another crucial feature of affordances, related to their very nature of being merely possible actions, is missed by STAP. Affordances, although relative to some characteristics, dispositional properties or potential behaviours of the individual (what ecological theorists call his "effectivities"), are not conditioned in their existence by the actual realizability or even availability of those behaviours ${ }^{2}$. Afforded actions - e.g., climbing stairs - have the subject's effectivities in their conditions of actualisation: they are only possible given those effectivities (Turvey, 1992), but they remain possible even if such conditions are not immediately fulfillable. Stairs afford climbing, even if I cannot climb them right now ${ }^{3}$. They afford climbing for systems able to climb them in situations where a climbing behaviour is actualizable. In short, affordances refer to "could", not to "can" actions: the resources that the environment offers exist - and can therefore be perceived - whether or not currently exploitable by the individual ${ }^{4}$.

\footnotetext{
${ }^{2}$ To the extent of my knowledge, this point is not highlighted as such by Gibson and his followers. In my opinion, it is nevertheless in line with their claims.

${ }^{3}$ Even more radically, one could defend with Michaels (2003) - and against authors as Kadar \& Shaw (2000) - that the affordances we perceive do not have to possess any counterpart in our own behavioural abilities.

${ }^{4}$ This claim probably does not apply to all kinds of affordances. For instance, it could be stated that an affordance as reachability of objects necessarily refers to the possibility of reaching the objects here and now, given the relative position of our body and the reach of our arm. Current exploitability would be in
} 
Why is this conception of affordances not compatible with the claim made by STAP supporters? The simulation-based affordance perception process described by STAP is clearly centred on the current characteristics, situation and dispositions of the individual performing it. Motor simulation is used to determine whether an action is feasible under the conditions specified by the current input (e.g., optic signal), given the current state of the body (e.g., the position of the arm), not whether it is feasible in itself or, say, in other specified circumstances. Therein lies the problem: a process determining if the action of making use of an object is feasible in current circumstances cannot decide whether this object has or has not the related affordance. As explained above, its affordances do not depend on what can be done right now. Motor simulation thus appears inappropriate to mediate perceptual access to affordances. The only thing simulation can do is to determine whether a specified action taking advantage of a specified affordance will be a success or a failure given current circumstances.

Supporting this view, recent studies have shown that the observation of graspable objects triggers activity in the motor cortex only when objects fall within the reaching space of the subject, suggesting that motor simulation is not involved for objects located too far to be grasped (Cardellicchio et al. 2011; see also Costantini et al., 2010, 2011). Yet this is not because the object is beyond reach that it is not graspable and perceived as such. From the moment that an object has suitable properties - e.g. size, form, material - it affords grasping, whether within or beyond reach.

For the sake of clarity, two kinds of "potential actions" consequently need to be distinguished when analysing perceptual access to so-called affordances: (a) actions that are possible in principle with the object (for a referent system), even if such actions are currently unavailable (the cup is graspable even if I am too far to grasp it); (b) actions that are currently feasible (or assumed to be) given our position and state (the cup, which is located within the reach of my arm, can be grasped). The latter kind can be regarded as a subset of the former.

Although necessary to delineate the exact scope of STAP, the previous criticism, however, remains of limited impact, when taken in isolation. Because it foremost deals with the meaning of the affordance concept, revising or simply challenging this meaning is sufficient to overcome it. One may in particular reject the previous considerations precisely because they promote a conception of affordances too independent of the situation, needs, goals, and mental states of the individual. After all, if, as stated by Gibson, affordances are not intrinsic physical properties, but relative to a system able to exploit them, should we not hold that they only exist when the circumstances for their exploitation are met, i.e. when they are currently feasible? What is the point in claiming that the cup is catchable "in principle", if it is catchable only when located at the correct height and grasp distance, and because I have hands to catch it? We could however expatiate on these issues for a long time, each of the two perspectives described having advantages and disadvantages (Michaels, 2003). The only thing we need to acknowledge at this step is that "possible" and "feasible" actions are two distinct categories, and that STAP probably exclusively covers the latter.

that case a constitutive component of the affordance. But this is clearly not the case for other types of affordances: a given solid surface affords support for standing and walking even if I am not in position to use it or - more radically - even if I am, for any reason, unable to use it (I might be paralysed). For the present purposes, this question can however remain open. 


\section{Insights from robotic research}

Another set of arguments challenging STAP hypothesis can be drawn from the robotic research literature. As mentioned in the introduction, STAP is not only defended in the field of neuropsychology, several attempts have been made to use the simulation framework to provide artificial systems with categorization skills supporting anticipation of behavioural possibilities afforded by objects (Möller, 1999; Ziemke el al., 2005; Hesslow \& Jirenhed, 2007; Hoffman, 2007; Erdemir et al., 2008; Schenck, 2009; Schenck et al., 2012). Assuming that 'mental imagery could be the basis for the detection of affordances in visual perception', e.g. Schenk et al. (2012) have used motor simulation algorithms to enable a mobile robot equipped with a camera to distinguish between types of obstacle arrangements: pass-through-able versus non-pass-throughable corridors (dead ends).

The success of STAP in the field of robotics is easily explained: building robots able to detect affordances would have indisputable practical benefits, e.g., for army or space clones design ${ }^{5}$. But application issues are not the only reason why STAP hypothesis is attractive for roboticists. The idea, which was first formulated by early AI theorists, like H.A. Simon (1969) and H.L. Dreyfus (1972), is now well known: artificial intelligence systems design can be considered an empirical method to test theoretical hypothesis on the nature and mechanisms of cognition. If you can build a robot whose behaviour relies on motor simulation and which behaves as living systems perceiving affordances behave, you will bring empirical support to the thesis that motor simulation is the kind of information processing mechanism such systems use to detect affordances.

However, as will be shown hereafter, achievements of roboticists are far from providing such support to STAP, quite the contrary in fact. This is not to say that engineering failures cast doubt on the theoretical relevance of STAP. Building machines reproducing natural cognitive processes can fail (or succeed) for a lot of extratheoretical reasons. As far as I know, attempts of STAP roboticists were in fact rather successful. The point I want to make is somewhat different: the attempts of roboticists to build machines able to perceive affordances challenge the claims made by STAP because they highlight concretely some implementation problems such hypothesis is faced with. This is the reason they are considered in this paper, regardless of their failure or success.

\subsection{Motor simulation is neither motor imagery nor sensorimotor emulation}

Before discussing the achievements of roboticists, some terminological remarks on the meaning of the concepts of (a) mental imagery, (b) motor simulation and (c) sensorimotor emulation, are necessary. Indeed, most researchers in robotics confound motor imagery, which is a kind of mental imagery, with motor simulation, and they talk of motor simulation when they think of sensorimotor emulation.

(a) As the name implies, mental imagery is an imagery process, and is consequently in a way or another related to images. This is not to say that it is an intrinsically visual process: mental imagery can clearly encompass the contribution of other modalities: you can imagine you hear a sound or feel something by touch (for recent reviews, see Iachini, 2011; Madan \& Singhal, 2012). In principle, we could assume that such a process is either explicit, for instance when you consciously imagine you rotate your hand to catch an object, or implicit, i.e., is not accompanied by a conscious display of the events, objects or states of affairs being represented. Most STAP roboticists and

\footnotetext{
${ }^{5}$ For a recent review of the use of the concept of affordance in robotics, see Sahin et al. (2007).
} 
authors such as Grush (2003) make use of the notion of motor or mental imagery to refer to robotic simulation processes, which, as far as we can judge, are not accompanied by awareness. But for sake of precision it is probably better to hold that imagery involves by definition a conscious experience: if there is no awareness of the process being simulated, it is simply not imagery. To say it metaphorically, an image needs a screen to be displayed. In the rest of the paper, we will consequently keep the concept of mental imagery for conscious processes of imagining something (i.e., imagination in the usual sense).

(b) Motor (or action) simulation refers to a quite different process, namely a dynamic representation of body activity and interactions. It consequently involves a model of the body, for instance its biomechanical structure, its capacities and the energetic cost of actions, and possibly a functional representation of tools or other artefacts and external resources we can use to reach a goal. Unlike mental imagery, motor simulation is not necessarily a conscious process. According to most neuroscientists, motor simulation plays its role in motor cognition in a purely implicit way (Parsons, 1994; Decety, 1996b; Jeannerod \& Frak, 1999). You can be aware of the motor simulation process being engaged (more precisely: motor simulation can have a conscious output). This is typically the case in mental rotation tasks that are body-centred: you can imagine yourself rotating your hand. You can also imagine that you use a tool to do something or that you walk from one place to another. However, such experience probably implies some additional processes compared with purely implicit motor simulation (basically the mechanism making us aware of the simulation performed). It is consequently better to use the concept of motor imagery - a kind of mental imagery process - to refer to the former.

(c) Sensorimotor emulation is still a different thing. It relates to the anticipation of the changes in the sensory input that a given motor action will (or at least should) lead to, e.g., the optic expansion flow occurring during our ambulatory movement or the displacement of a given pattern in the optical field following eye movements. It is a pure "calculation" process, and does not need any consciousness or even intentional (in the broad sense of "aboutness") component. Emulators are forward models, and a lot of artificial intelligence systems make use of such models to regulate their activity, for instance systems for ship navigation or robotic arms control (Grush, 2003; Schenck, 2009). Sensorimotor emulation has thus a quite different meaning than motor simulation. In a way, sensorimotor emulation is always involved in motor simulation (as well as in mental imagery: in mental rotation tasks, one can imagine what modifications in the visual appearance of the object will be induced by its rotation), but motor simulation works at a more macroscopic and sophisticated level of representation. What is simulated by motor simulation processes has a more "concrete" format than raw sensory input or signals: motor simulation deals with kinetic components of actions and spatial and dynamic features of body and objects (Grush, 2004).

Although they are frequently confused, motor simulation must consequently be clearly distinguished from mental imagery as well as sensorimotor emulation: (i) Unlike mental imagery, which is explicit and does not necessarily involve a representation of body activity (you can rotate an object "in your head" to find its symmetric axis - a classical task in mind games - without imagining that you use your hands), motor simulation can be - and is most of the time - implicit and always works with models of the body. (ii) Unlike sensorimotor emulation, which refers to a blind calculation process of sensory inputs, motor simulation works with a more sophisticated representation format (broadly speaking: perception, not sensation), and the simulated action sequence can be consciously experienced (we talk in this case of motor imagery). 


\subsection{Sensorimotor emulation is not sufficient to predict actions potentiated by objects}

On the basis of this conceptual clarification, it must first be emphasised that STAP hypothesis refers in principle to motor simulation, not to mental imagery. Obviously, you don't have to explicitly imagine yourself sitting on this chair to perceive it affords "sittability": most often you "see" this "sittability" (or you "know" the chair is "sittable": you take for granted that it could be used to sit) without even paying attention to it. Mental imagery could also lack some characteristics of action representation that seem essential to build realistic representations (i.e., to represent feasible, not fanciful actions), as sensitiveness to biomechanical constraints. As outlined by Johnson (2000), 'transformations of visual mental images are well known to follow the shortest path irrespective of such boundaries' 6 .

The same kind of remarks applies in theory to sensorimotor emulation. If an information processing mechanism must be able to determine what actions are feasible in the environment, it cannot merely emulate the sensory input changes that should be induced by the motor command. Yet, as mentioned in the previous section, motor simulation and sensorimotor emulation are frequently confused by STAP supporters, especially in the robotics literature. Schenck (2009) and Schenck et al. (2012), for instance, claim that affordance perception relies on emulation mechanisms predicting the sensory consequences of motor commands (what they improperly call mental imagery or mental simulation process), and that such mechanisms can empower robots with the ability to perceive what their environment affords, for example dead end corridors, and regulate accordingly their behaviour: robots do not have to enter such corridors to "understand" that they will confront with a dead end; by processing the current input of the camera with emulation algorithms, they can directly categorize the corridor structure as not-pass-through-able.

However, predicting the sensory consequences of a given motor command (or sequence of motor commands, whatever), i.e. the changes in sensory input, say in the optic array, that this command should produce, is clearly not sufficient to assess the feasibility of a given action in a given context. Actions that can be undertaken with a given object cannot be predicted on the sole basis of the anticipation of changes in sensory input. As Cisek (2007) explains, 'specification of actions [...] requires information about the spatial relationships among objects and surfaces in the world, represented in a coordinate frame relative to the orientation and configuration of the animal's body'. To represent an action sequence as grasping an object or using a wrench, the system must take into account its spatial, dynamic and physical properties, for instance the position in space and the velocity of the arm during the different phases of the action, or the muscular effort that should be provided to master the inertial properties of the limb or the weight of the tool. As we have seen in the previous section, only motor simulation represents such properties when playing in advance action sequences. Only motor simulation has consequently the representational resources to

\footnotetext{
${ }^{6}$ This claim could be discussed. Several studies have shown that some of the constraints on overt action were preserved in motor imagery, as Fitt's law or time and energetic constraints on body displacement (Decety \& Jeannerod, 1996). Decety \& Sommerville (2007) thus claim that 'one reason why motor imagery allows us to plan actual actions is that the constraints of the physical world shape our imagery in a manner similar to how they shape our actions.' However, those observations do not demonstrate that mental - or motor - imagery necessarily complies with such constraints. After all, one can imagine oneself performing "impossible" actions, as flying, running as fast as a car or lifting mountains, or one can e.g. imagine that one's arm can stretch to catch distant objects as in Witt \& Profitt (2008)'s study.
} 
predict action feasibility. Sensorimotor emulation precisely works at the sensorimotor level, where the information necessary to evaluate such feasibility is not available.

\subsection{Predicting actions in an uneventful microworld is not the same as in an unpredictable reality}

Another kind of difficulty STAP is faced with is related to the problem of the computational resources that should be available to simulate affordances related actions. This can be understood from the reflections proposed by Hesslow (2002). According to Hesslow (2002), motor simulation can be used to represent and assess the feasibility of extended behavioural sequences. 'Once the mechanism of anticipation is in place, perceptual activity generated by a simulated action can serve as a stimulus for a new response, and so on [...], thus enabling long chains of simulated responses and perceptions.' The problem-solving process involved in tasks as the "Tower of London" is a typical example of such a mechanism. One may however be sceptical about the extension of this hypothesis to ecological situations of interaction with the environment. The real world is far more complex than the "Tower of London" microworld, where possible configurations and actions can be counted on the fingers of one hand. Obviously, the number of iterative steps the simulation process can achieve should be drastically reduced because of the combinatorial explosion of possibilities implied by the unpredictability of the real environment. Admittedly, in a sense this unpredictability only applies to details; the overall scenario is usually uneventful. But this is not this scenario that the simulation process must anticipate. To determine whether a given action is feasible in the situation, e.g. grasping an object, the motor simulation process must reflect accurately the properties of the body, its position in space and grasping capacities, as well as the current characteristics of the object in the environment: if not, the prediction will prove wrong.

In fact, even in ultra-simplified microworld situations, the process of anticipation quickly faces with computational limits. This is something AI theorists such as John McCarthy and Marvin Minsky have widely shown while studying chess. The computational resources of a Deep blue are required when it comes to predicting the possible game configurations and evaluate their interest several steps ahead. In one way or another, it is possible to anticipate the possibilities made available by a complex situation. This is what we all do constantly. And for this, we probably make use of 'internal models' of the behaviour of agents and objects, including a so-called naive physics. But such a prediction relies on processes much more (or maybe less) complex than just emulating sensory consequences of actions, typically what AI researchers call heuristics: clever methods to reduce drastically the search space.

Schenck et al. (2012)'s claims about the function motor simulation plays in action anticipation meet the same kind of difficulties. The authors oppose the motor simulation-based control of behaviour to the classical computo-symbolic approach in $\mathrm{AI}$, and claim that the latter is successful in well-controlled task domains with a restricted or well-known set of objects, but lacks the flexibility and adaptivity of human vision'. The problem is that the simulation-based approach they promote suffers from precisely the same limitations. As long as the robot operates in a microworld where the possible configurations of the environment and the possible behaviours are in limited number and known in advance, the actions that can be realized given the current state of affairs can be predicted using "motor simulation". Things are nevertheless getting more complicated if the environment gets more complex and the action repertoire broader. A reliable anticipation of the consequences of actions certainly remains possible when only two alternative motor commands must be tested, as in the example proposed by 
Schenck (2009) (although one quickly faces with a combinatorial explosion when pushing the simulation several steps in the future). But if the system only has to decide between two motor commands, it means that what it plans to (or can) do in the environment is almost already decided. The behavioural repertoire of a human being in ecological context is far more complex, as the motor programs that enable their achievement.

The proposition that perceptual access to affordances is mediated by an action simulation mechanism is thus confronted with a computational reality principle. As long as a limited number of actions have to be simulated to determine what environment structures make it possible to do, the idea remains plausible, but if the repertoire of actions potentially relevant in a situation is broader (as we can assume it is the case in humans in ecological context), such a mechanism is no more sufficient.

Möller (1999) was aware of that problem: 'A drawback of the anticipation approach is the time required for the selection of appropriate actions: the agent has to simulate a number of sensorimotor sequences in order to find a sequence with a positive value that is suitable for execution.' Hoffmann (2007) arrives at a similar conclusion: the kind of recursive search used in simulation algorithms 'is computationally expensive and prone to prediction errors for large search depths. Thus, sensorimotor anticipation by itself cannot generate a behavior from sensory input by testing all possible movement variants. Instead, other processes need to trigger a suitable movement plan.'

Notwithstanding, for the authors this problem is partially solved if the system is provided with a mechanism enabling to associate the current situation (which acts, via the sensors, as input for the simulation process) and typical reactions. 'That way, the search space for the motor commands is restricted and the effort in time is reduced: with features indicating an obstacle on the right, the simulation will preferably test movements to the left' (Möller, 1999). One can only agree with such proposition, which indeed seems to correspond to a quite faithful description of our experience and behaviour in the real world: the potential actions we envisage in a given situation always appear constrained by our habits and some contextual knowledge of the situation and place where we are. The problem is that such considerations also imply a drastic weakening of the STAP hypothesis. If the system already knows what the typical ways to react in a given situation are, the biggest part of the path to explain how we get knowledge of what can be done with surrounding structures (or cannot be: passing through obstacles) has already been covered.

What previous considerations thus suggest is that the mechanisms described by STAP already presuppose some perceptual knowledge of the action possibilities made available by the environment. This is the only way to avoid an infinite regression. STAP posits that access to affordances is enabled by a simulation process testing the feasibility of the afforded behaviour. The problem is however: how to determine, for a given object $O$, the actions (or, at another level of description, the motor programs) whose achievement should be simulated? If the system already knows those actions, does it not mean that it (at least partially) knows which affordances are potentiated by the object?

Thinking about it, the solution to that Escherian problem is quite obvious: a first layer of affordances must already be available for the motor simulation mechanisms to work. This point is evident once recognized that motor simulation does not deal with (intrinsically) possible actions, but with (here and now) feasible actions. To determine if an object $O$ supports the realization of a behaviour $C l$ of a behavioural repertoire $\{C 1$, ..., $C n$ \}, for instance if an object can be grasped using a thumb-index precision grip, in a way or another the object $O$ must already have been apprehended as belonging to the 
category of graspable objects, that is to say the objects which support in principle the achievement of such behaviour (solid objects within a certain range of sizes).

\section{Affordances we are perceptually aware of do not reduce to affordances we pay attention to}

STAP's explanation of our perceptual access to affordances is also insufficient in different respects when taking into account the phenomenological properties of this access.

A first argument in this regard, still related to the problem of computational resources discussed above, is that the affordances we are aware of at every moment are far too many for their realization to be simulated by the brain, no matter if in series or parallel. This point can be understood if we distinguish between central and peripheral perceptual awareness.

In empirical studies described by STAP supporters (e.g. De'Sperati \& Stucchi, 1997, 2000; Tucker \& Ellis, 1998, 2001, 2004; Ellis \& Tucker, 2000; Coello \& DelevoyeTurrell, 2007; Witt \& Proffitt, 2008), the individual is usually confronted with one specific object he has to categorize or toward which he must direct an action. But in ecological circumstances - typically in the rooms of a house - this is not one, but dozens or even hundreds of objects we perceive. Certainly, at every moment, our attentional focus is directed toward one single object, set of objects or process. We have nevertheless a peripheral perception of other surrounding objects. We are peripherally aware of their presence. And we implicitly know what they can be used for. In a way, such awareness even applies to objects that are outside our peripheral perceptual field, i.e., objects that do not appear but are nonetheless participating in the situation. I do not see the scissors lying on the desk behind my back. But I rely on their availability: the cutting action they enable belongs to my behavioural field. The same applies for any usable object located in our close environment, i.e., which is within range. We know that objects are available if we need them, and we know (more or less) where they are stored and how to find them. Without such peripheral knowledge, we could not know that we must go into the kitchen to get a glass of water, but into the living room for a glass of scotch. Our perceptual awareness of what stands around us would reduce to the sole part of the environment we are currently paying attention to. If, as stated by Garbarini \& Adenzato (2004), 'only by virtually executing the action can we understand the relational significance of the object, i.e., the affordance it offers', it is consequently not one, but hundreds of action sequences that have to be processed to make us perceive the affordances our environment makes available.

The problem of STAP is that it seems to reduce the perceptual field to the attentional field, or at least to hold that only the objects to which our attention is turned at time $t$ are perceived as affording something, objects belonging to what Searle or other researchers call peripheral awareness being not considered in this manner (if having any perceptual reality). But just as there is a central and a peripheral vision, our perceptual awareness is divided into a central and a peripheral field. We have the impression that we are only aware of the portion of reality that is under attentional spotlight, but this is a mistake: we are constantly aware of the periphery, and this context or background contributes to determine the content of that which is the subject of our explicit perception ${ }^{7}$. When I am

\footnotetext{
${ }^{7}$ This point was especially made by Husserl: each perceived object is given with an external horizon, it is accompanied by a co-perception of other present objects and to which it is possible to turn. See e.g. Husserl (1950): §19.
} 
visually aware of the coffee table in the living room, I do not perceive an object insulated from the rest of the world. The table is precisely perceived as being in the living room, which is in the house, which is in the city. The house, with all the objects and related affordances - it contains, is, so to speak, co-perceived when I see the table. The awareness - more or less implicit - of being located in a certain place of the network of familiar places the world consists of encompasses an awareness - more or less implicit - of the behavioural resources made available by that place. I know I am at the office, in a restaurant, in the subway, on the street or at home in the living room. The "concept" of living room as it occurs in my experience of being somewhere includes the objects this place generally contains, with the actions they potentiate, somewhat in the style of Marvin Minsky's frames ${ }^{8}$.

One may retort that strictly speaking we only perceive the affordances in the beam of our attention at time $t$, that such peripheral awareness is illusory, and stems from our certainty of being able to turn our attention to the elements of the periphery ${ }^{9}$. But in this case how to explain that we know where to turn our attention? Most importantly, how to explain that when planning our behaviour we take into account opportunities for action provided by those structures that are not subject to direct perception? As Searle (2007) explains, the proof that such peripheral elements 'are a part of my conscious field is that I can at any moment shift my attention to them. But in order for me to shift my attention to them, there must be something there which I was previously not paying attention to which I am now paying attention to.'

\section{Motor simulation is not requested for affordances already specified by the structure of the perceptual field}

Next, one may be dubious about the interest - and thus cognitive reality - of simulating an action to evaluate whether it is afforded by the environment, when the spatial layout, to reuse Gibson's expression, is sufficient to get such knowledge.

Visual distance perception is a good illustration. In visual experience, the environment is organized along a gradient of distance. This gradient enables to locate objects in relation to our body, and assign to each a value in terms of accessibility: near means accessible, far means not directly accessible. This accessibility can for instance be specified in terms of walking time. Through the gradient of distance, we are thus immediately aware of what we can or cannot do. This is, so to say, explicit in the appearing structure of the spatial layout.

Now, psychologists have long shown that the configuration of this gradient proceeds from the action of several static or dynamic optical variables (binocular disparity, height in the visual field, shadows and texture gradients of surfaces, relative sizes of objects, parallax, etc.). These variables together contribute to define the position of objects in the distance (Cutting \& Vishton, 1995). Obviously, this mechanism does not need to simulate the action of accessing objects to work. You do not have to simulate the action of reaching for perceiving an object at ten meters as being beyond reach. The same goes for the reachability of nearby objects. The gradient of distance enables a direct apprehension of the (in)accessibility of surrounding structures.

This does not imply that motor simulation cannot be involved in the visual perception of reachabiliy. But it will only intervene when other - more direct - reachability

\footnotetext{
${ }^{8}$ Based on Heidegger's phenomenological analysis of our everyday coping with objects, Turner (2005) makes a similar claim: affordances participate in a network and we never perceive them in isolation.

${ }^{9}$ A similar argument was used by O'Regan \& Noë (2001) against internalist approaches of perception.
} 
estimation mechanisms lose their reliability, e.g. when an object to be grasped is located at the boundary of the arm reachable space. Supporting this view, Coello et al. (2008) have shown that transcranial magnetic stimulation of the motor cortex only interferes with judgments of reachability for targets located at the limits of reachable space, which tends to demonstrate 'that action simulation would be required mainly when the determination of what is reachable becomes ambiguous'. The fact that the time required for judgments increases substantially for visual targets located in this critical area further supports this hypothesis (Bourgeois et al., 2009).

The same conclusion can be drawn from the analysis of the visual perception of the solidity or impenetrability of objects. Seeing something as a solid object, seeing that there is an impenetrable structure in front of us: a table for example, means ipso facto taking for granted that some behavioural possibilities are available (we can put objects or sit on its surface), while others are neutralized (we have to walk around the table to pass). However, as in the case of distance, it is highly unlikely that the perception of such action possibilities and impossibilities relies on a motor simulation mechanism. Obviously, I do not have to simulate the contact between the object and my body to anticipate its impenetrability and related affordances. What would be the point? As shown by Gibson (1958), the optical array is already a reliable resource to specify solid surfaces. Moreover, impenetrability is not something one anticipates (except in situations of reduced vision, as in the fog), it is something one sees. As the saying goes, seeing is believing, and seeing something as solid means precisely counting on the possibilities potentiated by the solidity of the object, taking for granted their availability.

Of course, the optical field taken as such is not sufficient to convey knowledge of what can be done in the environment. An object will be reachable only if it is located within the reach of a system possessing a reaching capacity. 'Reachability is codetermined by the characteristics of the object and those of the actor in terms of his/her capacity for action and situation in the environment' (Rochat et al., 1999). Similarly, a solid object will only afford contact for a system having an impenetrable body. Yet, our body can serve as a metric to calibrate the optical field without the mediation of motor simulation (see e.g. Mark, 1987; Warren \& Whang, 1987). For reachability, the only requisite is to "remember" the reach of our repeated arm movements using optical cues, i.e. to use the grasping distance as defined in the optical field as a metric to calibrate visual distances ${ }^{10}$ (the same mechanism could be used to calibrate the size of objects using the metric of our hand grip span). For solidity, it is to associate some optical invariant, as the opacity of surfaces, with the property of impenetrability.

A similar claim could be made for affordances which do not seem to be directly accessible through spatial layout. For example, consider a lake covered with ice and the perception that its surface is strong enough to support our body: we perceive the surface as affording standing and walking. Do we need to simulate the activity of standing and

${ }^{10}$ Coello \& Delevoye-Turrell (2007) have observed that when modifying the apparent extent of the reaching capacities of subjects by providing them with a fake visual feedback of the distances their arm can reach, the space they perceive as reachable runs a constriction. The authors explain this result by claiming that the perception of objects' reachability relies on a motor simulation process using internal models of the biomechanical structure and action capability of the body: the visual bias alters the model of the body used to simulate actions, and thus alters the objective distance to which objects must be situated to be perceived as affording reaching. However, this observation does not prove that motor simulation is necessary for reachability perception, and it does not even prove that motor simulation is responsible of this distance constriction phenomenon. What this study shows is only that our perception of reachability is calibrated on our action capacities, and that those capacities play a metric role in the determination of visual distance. 
walking on the lake (using internal models of our body's weight and naive physics of the ice's resistance) to perceive such affordances? The idea seems absurd. It is clear that other mechanisms support perceptual access to such affordances, typically simple visual cues: the aspect of the ice, its colour, transparency, if it seems thick, if it is already a little melted, and even more importantly, if others are already standing on it.

\section{Conclusion: motor simulation does not explain but presupposes affordance perception}

What previous considerations demonstrate is that motor simulation mechanisms, as described by STAP supporters, (1) only apply to objects we are explicitly paying attention to, not to all objects and related affordances we are aware of at time $t$; (2) can only work if some perceptual knowledge of the action possibilities made available by those objects is already available. Far from explaining affordance perception, the motor simulation framework thus presupposes such perception.

It does not mean that motor simulation has no role to play in the cognitive process enabling the perception of action possibilities made available by the environment. Motor simulation is probably centrally involved when an explicit evaluation of action feasibility is engaged, and in critical situations, e.g. when an object to be grasped is located at the limits of reachable space (Coello et al., 2008).

The investigation proposed in this paper also suggests that several categories of actions need to be distinguished to clarify the problem of how we perceive what surrounding objects afford: $(i)$ actions that are in principle possible given the properties of the object (this cup is graspable for a standard human hand); (ii) actions that are immediately feasible, i.e. feasible here and now given my state and situation relative to the object (the cup within my arm's reach is graspable); (iii) actions that are mediately feasible, i.e. which are only feasible if other actions are first performed (the cup beyond my arm's reach is graspable only if I first move closer to it); (iv) actions that are potentially relevant in the situation, given my current objectives, capacities, resources (grasping this cup is relevant whenever I plan to drink my coffee). Perceptual awareness of what we can or cannot do in the environment in a way or another involves all those action categories. But our access to each certainly relies on quite different mechanisms.

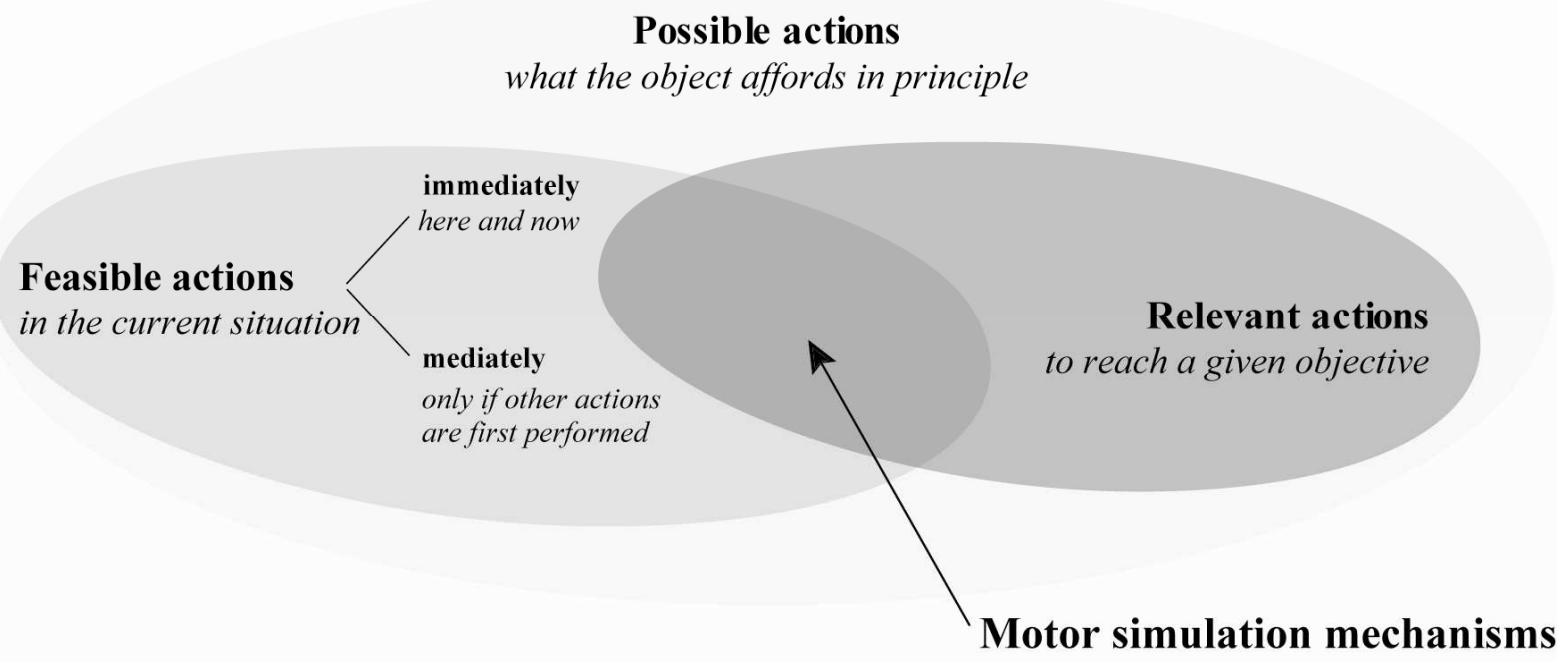

Figure 1 - Types of actions processed by the mechanisms supporting our perceptual awareness of affordances. 
If motor simulation plays any role here, it can only be in the mechanisms assessing if a given (possible) action supported by a given environment structure is feasible in the situation, and it seems reasonable to assume, in line with Möller (1999) and Hoffmann (2007), that motor simulation will only be engaged to evaluate the feasibility of actions considered as contextually relevant (see Cisek, 2007, for a similar claim). Those two categories of action have to be clearly distinguished. Determining what is feasible (immediately or with a delay) is totally different from determining what is potentially relevant in a given situation. The relevance of an action above all depends on its capacity to fulfil some specified objectives (i.e., to be the means to achieve a goal). Besides, the fact that something cannot be done does not mean it is not relevant. Not currently feasible actions can be totally relevant to reach one objective, and taking into account such actions could be important in deciding what other resources than those currently available shall be provided to this end (Michaels, 2003).

Strictly speaking, motor simulation thus cannot provide knowledge of what is possible to do with objects (affordances). The best it can tell is whether an action already identified as possible and relevant can be performed in present circumstances.

\section{Acknowledgments}

I am grateful to the reviewers of Adaptive behaviour for their insightful comments on earlier versions of this paper. I also express my gratitude to Sajjad Hussain and JeanBaptiste Guignard for proof-reading this paper.

\section{Funding}

This research received no specific grant from any funding agency in the public, commercial, or not-for-profit sectors.

\section{References}

Bourgeois, J., Bartolo, A., \& Coello, Y. (2009). Sensorimotor adaptation to a biased feedback modifies the visual perception of peripersonal space. Perception, 38, ECVP Abstract Supplement, 67.

Caligiore, D., Borghi, A. M., Parisi, D., \& Baldassarre, G. (2010). TRoPICALS: A computational embodied neuroscience model of compatibility effects. Psychological Review, 117(4), 1188-1228.

Cardellicchio, P., Sinigaglia, C., \& Costantini, M. (2011). The space of affordances: A TMS study. Neuropsychologia, 49(5), 1369-1372.

Chemero, A., \& Turvey, M. T. (2007). Gibsonian affordances for roboticists. Adaptive behaviour, 15(4), 473-480.

Cisek, P. (2007). Cortical mechanisms of action selection: The affordance competition hypothesis. Philosophical Transactions of the Royal Society B., 362, 1585-1599.

Coello, Y., \& Delevoye-Turrell, Y. (2007). Embodiment, spatial categorisation and action. Consciousness and Cognition, 16, 667-683.

Coello, Y., Bartolo, A., Amiri, B., Devanne, H., Houdayer, E., \& Derambure, P. (2008). Perceiving what is reachable depends on motor representations: Evidence from a transcranial magnetic stimulation study. Plos One, 3(8), 1-12. 
Costantini, M., Ambrosini, E., Tieri, G., Sinigaglia, C., \& Committeri, G. (2010). Where does an object trigger an action? An investigation about affordances in space. Experimental brain research, 207(1), 95-103.

Costantini, M., Ambrosini, E., Scorolli, C., \& Borghi, A. M. (2011). When objects are close to me: Affordances in the peripersonal space. Psychonomic bulletin \& review, 18(2), 302-308.

Craik, K. (1943). The nature of explanation. Cambridge: Cambridge University Press, 2010.

Cutting, J. E., \& Vishton, P. M. (1995). Perceiving layout and knowing distances: The interaction, relative potency, and contextual use of different information about depth. In W. Epstein \& S. Rogers (Eds.), Perception of space and motion. (pp. 69-117). San Diego, CA: Academic Press.

Decety, J. (1996a). The neurophysiological basis of motorimagery. Behavioural Brain Research, 77, 45-52.

Decety, J. (1996b). Do imagined and executed actions share the same neural substrate? Cognitive Brain Research, 3, 87-93.

Decety, J., \& Jeannerod, M. (1996). Fitts' law in mentally simulated movements. Behavioral Brain Research, 72, 127-134.

Decety, J., \& Sommerville, J. (2007). Motor cognition and mental simulation. In S.M. Kosslyn, \& E. Smith (Eds.), Cognitive Psychology: Mind and Brain. (pp. 451-481). New York: Prentice Hall.

Decety, J., \& Stevens, J. (2009). Action representation and its role in social interaction. In K.D. Markman, W.M.P. Klein, \& J.A. Suhr (Eds.), The Handbook of Imagination and Mental Simulation. (pp. 3-20). New York: Psychology Press.

Delevoye-Turrell, Y., Bartolo, A., \& Coello, Y. (2010). Motor representations and the perception of space: perceptual judgments of the boundary of action space. In N. Gangopadhyay, M. Madary, \& F. Spicer (Eds.), Perception, Action, and Consciousness: Sensorimotor Dynamics and Two Visual Systems. (pp. 217-242). Oxford: Oxford University Press.

De'Sperati, C., \& Stucchi, N. (1997). Recognizing the motion of a graspable object is guided by handedness. Neuroreport, 8, 2761-2765.

De'Sperati, C., \& Stucchi, N. (2000). Motor imagery and visual event recognition. Experimental Brain Research, 133, 273- 278.

Dreyfus, H. L (1972). What computers can't do: A critique of artificial reason. New York: Harper and Row.

Ellis, R., \& Tucker, M. (2000). Micro-affordance: The potentiation of components of action by seen objects. British Journal of Psychology, 91, 451-471.

Ellis, R., Swabey, D., Bridgeman, J., May, B., Tucker, M., \& Hyne, A. (2013). Bodies and other visual objects: the dialectics of reaching toward objects. Psychological Research, 77, 31-39.

Erdemir, E., Frankel, C. B., Kawamura, K., Gordon, S. M., Thornton, S., \& Ulutas, B. (2008). Towards a cognitive robot that uses internal rehearsal to learn affordance relations. Proceedings of the IEEE/RSJ International Conference on Intelligent Robots and Systems (pp. 2016-2021), September 22-26 ${ }^{\text {th }}$, Nice, France.

Gallese, V., \& Goldman, A. (1998). Mirror neurons and the simulation theory of mindreading. Trends in Cognitive Sciences, 2(12), 493-501. 
Gallese, V. (2000). The inner sense of action: Agency and motor representations. Journal of Consciousness Studies, 7, 23-40.

Garbarini, F., \& Adenzato, M. (2004). At the root of embodied cognition: cognitive science meets neurophysiology. Brain Cognition, 56, 100-106.

Gibson, J. J. (1958). Visually controlled locomotion and visual orientation in animals. British Journal of Psychology, 49, 182-194.

Gibson, J. J. (1986). The ecological approach to visual perception. Hillsadle, N.J.: Lawrence Erlbaum Associates.

Goldman, A. I. (2005). Imitation, mind reading, and simulation. In S. Hurley, \& N. Chater (Eds.), Perspective on Imitation, from Neuroscience to Social Science. (vol. 2, pp. 79-93). Cambridge: MIT press.

Goodale, M. A., \& Milner, A. D. (1992). Separate visual pathways for perception and action. Trends in Neurosciences, 15, 20-25.

Grush, R. (2003). In defense of some 'Cartesian' assumptions concerning the brain and its operation. Biology and Philosophy, 18(1), 53-93.

Grush, R. (2004). The emulation theory of representation: motor control, imagery, and perception. Behavioral and Brain Sciences, 27(3), 377-396.

Grush, R. (2007). Skill Theory v2.0: Dispositions, emulation, and the spatiality of perception. Synthese, 159(3), 389-416.

Hesslow, G. (2002). Conscious thought as simulation of behaviour and perception. Trends in Cognitive Sciences, 6, 242-247.

Hesslow. G., \& Jirenhed, D. A. (2007). Must machines be zombies? Internal simulation as a mechanism for machine consciousness. Proceedings of AAAI Symposium, Machine Consciousness and AI. November 8-11 ${ }^{\text {th }}$, Washington DC.

Hoffmann H. (2007). Perception through visuomotor anticipation in a mobile robot. Neural Networks, 20 (1), 22-33

Husserl, H. (1950). Cartesian Meditations: An Introduction to Phenomenology. The Hague: Martinus Nijhoff, 1960.

Iachini, T. (2011). Mental imagery and embodied cognition: A multimodal approach. Journal of Mental Imagery, 35, 1-26.

Jeannerod, M., \& Frak, V. (1999). Mental imaging of motor activity in humans. Current Opinion in Neurobiology, 9, 735-739.

Jeannerod, M., \& Gallagher, S. (2002). From action to interaction: An interview with Marc Jeannerod. Journal of consciousness studies, 9(1), 3-26.

Jeannerod, M. (1994). The representing brain. Neural correlates of motor intention and imagery. Behavioural and Brain Sciences, 17, 1987-2245.

Jeannerod, M. (2001). Neural simulation of action: A unifying mechanism for motor cognition. NeuroImage, 14, 103-109.

Jeannerod, M. (2003). Consciousness of action and self-consciousness: A cognitive neuroscience approach. In J. Roessler, \& N. Eilan (Eds.), Agency and Self-Awareness. (pp. 128-149). Oxford: Oxford University Press.

Jeannerod, M., Arbib, M. A., Rizzolatti, G., \& Sakata, H. (1995). Grasping objects: the cortical mechanisms of visuomotor transformation. Trends in Neurosciences, 18, 314320.

Johnson, S. H. (2000). Thinking ahead: The case for motor imagery in prospective judgments of prehension. Cognition, 74, 33-70. 
Johnson-Frey, S. H. (2004). The neural bases of complex tool use in humans. Trends in Cognitive Sciences, 8(2), 71-78.

Kadar, E., \& Shaw, R. E. (2000). Toward an ecological field theory of perceptual control of locomotion. Ecological Psychology, 12, 141-180.

Madan, C., \& Singhal, A. (2012). Motor imagery and higher-level cognition: Four hurdles before research can sprint forward. Cognitive Processing, 13, 211-229.

Mark, L. S. (1987). Eyeheight-scaled information about affordances: A study of sitting and stair climbing. Journal of Experimental Psychology: Human Perception and Performance, 13(3), 361.

Michaels, C.F. (2003). Affordances: Four Points of Debate. Ecological Psychology, 15(2), 135-148.

Möller, R. (1999). Perception through anticipation. A behavior-based approach to visual perception. In A. Riegler, M. Peschl, \& A. Von Stein (Eds.), Understanding representation in the cognitive sciences. (pp. 169-176). New York: Plenum Academic / Kluwer Publishers.

Negri, G. A. L., Rumiati, R. I., Zadini, A., Ukmar, M., Mahon, B. Z., \& Caramazza, A. (2007). What is the role of motor simulation in action and object recognition? Evidence from apraxia. Cognitive Neuropsychology, 24, 795-816.

O'Regan, J. K., \& Noë, A. (2001). What it is like to see: A sensorimotor theory of visual experience. Synthese, 129(1), 79-103.

Parsons, L. M. (1994). Temporal and kinematic properties of motor behaviour reflected in mentally simulated action. Journal of Experimental Psychology: Human Perception and Performance, 20, 709-730.

Pulvermüller, F. (2005). Brain mechanisms linking language and action. Nature Review Neuroscience, 6, 576-582.

Rochat, P., Goubet, N., \& Senders, S. J. (1999). To reach or not to reach? Perception of body effectivities by young infants. Infant and Child Development, 8(3), 129-148.

Schenck, W., Hasenbein, H., \& Möller, R. (2012). Detecting affordances by mental imagery. In A.G. Di Nuovo, V.M. De la Cruz, \& D. Marocco (Eds), Proceedings of the 12th International Conference on Adaptive Behaviour (SAB 2012), Workshop on Artificial Mental Imagery in Cognitive Systems and Robotics (pp. 15-32). August $27^{\text {th }}$, Odense, Denmark.

Schenck, W. (2009). Space perception through visuokinesthetic prediction. In G. Pezzulo, M. Butz, O. Sigaud, \& G. Baldassarre (Eds.), Anticipatory Behavior in Adaptive Learning Systems: From Psychological Theories to Artificial Cognitive Systems, Lecture Notes in Artificial Intelligence, 5499. (pp. 247-266). Berlin, Heidelberg, New York: Springer.

Searle, J. R. (2007). The problem of consciousness. In G. R. Bock, \& J. Marsh (Eds), Experimental and theoretical studies of consciousness, CIBA Foundation Symposium 174. Chichester, UK: John Wiley \& Sons.

Simon H. A. (1969). The Sciences of the Artificial. Cambridge: MIT Press.

Sinigaglia, C., \& Rizzolatti, G. (2011). Through the looking glass: Self and others. Consciousness and Cognition, 20, 64-74.

Tipper, S. P., Paul, M. A., \& Hayes, A. E. (2006). Vision-for-action: The effects of object property discrimination and action state on affordance compatibility effects. Psychonomic bulletin \& review, 13(3), 493-498. 
Tucker, M., \& Ellis, R. (1998). On the relations between seen objects and components of potential actions. Journal of Experimental Psychology: Human Perception and Performance, 24, 830-846.

Tucker, M., \& Ellis, R. (2001). The potentiation of grasp types during visual object categorization. Visual Cognition, 8, 769-800.

Tucker, M., \& Ellis, R. (2004). Action priming by briefly presented objects. Acta Psychologica, 116, 185-203.

Turner, P. (2005). Affordance as context. Interacting with computers, 17(6), 787-800.

Turvey, M. T. (1974). Constructive theory, perceptual systems, and tacit knowledge. In W. B. Weimer, \& D. S. Palermo (Eds), Cognition and the symbolic process. Hillsdale, N.J.: Lawrence Erlbaum Associates.

Turvey, M. T. (1992). Affordances and Prospective Control: An outline of the ontology. Ecological Psychology, 4, 173-187.

Ungerleider, L., \& Mishkin, M. (1982). Two cortical visual systems. In D. J. Ingle, M. A. Goodale, \& R. J. W. Mansfield (Eds.), Analysis of visual behaviour. (pp. 549-586). Cambridge: MIT Press.

Warren Jr, W. H., \& Whang, S. (1987). Visual guidance of walking through apertures: Body-scaled information for affordances. Journal of Experimental Psychology: Human Perception and Performance, 13(3), 371.

Wheeler, K., \& Bergen, B. (2010). Meaning in the palm of your hand. In S. Rice, \& J. Newman (Eds.), Empirical and Experimental Methods in Conceptual structure, Discourse, and Language. Stanford: CSLI.

Witt, J. K., Kemmerer, D., Linkenauger, S. A., \& Culham, J. (2010). A functional role for motor simulation in naming tools. Psychological Science, 21, 1215-1219.

Witt, J. K., \& Proffitt, D. R. (2008). Action-specific influences on distance perception: A role for motor simulation. Journal of experimental psychology: Human perception and performance, 34(6), 1479-1492.

Ziemke, T., Jirenhed, D.A., \& Hesslow, G. (2005). Internal simulation of perception: A minimal neuro-robotic Model. Neurocomputing, 28, 85-104. 